\title{
Characterization of multiple piezoelectric actuators for structural excitation
}

\author{
Robert L. Clark, Chris R. Fuller, and AI Wicks \\ Mechanical Engineering Department, Virginia Polytechnic Institute and State University, \\ Blacksburg, Virginia 24061
}

(Received 21 May 1990; revised 7 February 1991; accepted 27 February 1991)

The thrust of the present work is to analytically and experimentally study the response of a simply supported beam driven by multiple piezoelectric actuators in an effort to understand distributed excitation of the structure. The results indicate that the theoretical model provides the basis for a viable means of determining appropriate locations for piezoelectric actuators for exciting desired modal distributions in the structural response.

PACS numbers: $43.40 . \mathrm{Cw}$, 43.88.Fx

\section{LIST OF SYMBOLS}

Arabic

[A] matrix of measured acceleration

$C_{0} \quad$ piezoelectric strain-beam moment

coupling term

$D \quad$ flexural rigidity

$d_{31} \quad$ piezoelectric strain constant

$E \quad$ Young's modulus

$G_{x x} \quad$ input autospectrum

$G_{x y} \quad$ cross spectrum

$G_{y y} \quad$ output autospectrum

$h$ half-thickness of beam

$H \quad$ model for frequency response function

$K \quad$ geometric nondimensional parameter

$L \quad$ length of beam

$m \quad$ interface moments per unit length

$m^{\prime \prime} \quad$ mass per unit area of beam

$M \quad$ internal beam moments

$n_{d} \quad$ number of averages

$P \quad$ constitutive nondimensional parameter

$r \quad$ radius of curvature

$[S]$ transformation matrix of eigenvectors

$t \quad$ piezoelectric patch thickness

$u\left({ }^{*}\right) \quad$ step function

$V \quad$ input voltage

$w \quad$ beam transverse displacement

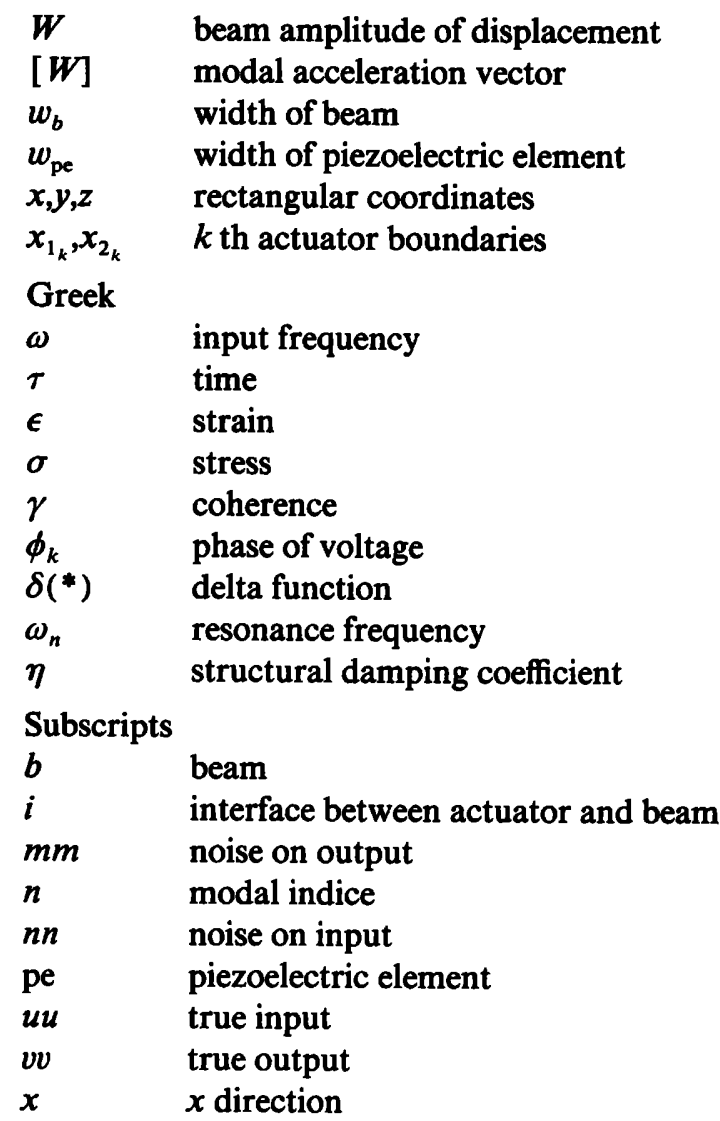

by Crawley and de Luis, an expression for the static and dynamic response of a cantilevered beam configured with two piezoelectric actuators was developed and experiments were conducted to evaluate this model. ${ }^{1}$

In this study, a theoretical model of a simply supported beam configured with multiple piezoelectric actuators is developed, and the experimental results are compared to the theoretical results at a variety of test frequencies both on and off resonance. The range of linearity for structural excitation was studied as well to determine the appropriate range for comparing experimental and theoretical results. To accomplish these goals, a simply supported beam was built and a 
pair of piezoelectric actuators were bonded to the surface of the beam. The beam was then driven at various test frequencies with different combinations of actuator forcing functions, and the modal response was measured.

Before attempting to study the aforementioned goals, a modal analysis of the beam was performed. Results from this experimental study can be compared to theoretical results obtained from an analysis of the structure based on assumptions of Euler beam theory. A complete outline of the theory behind this work is presented in Sec. I. A description of the hardware necessary to conduct the experiment follows with emphasis on fabrication of the beam and bonding of the actuator to the surface. After developing the background and necessary tools for analysis, the results are presented from the modal analysis, linearity test, and study of distributed excitation.

\section{THEORY}

\section{A. Beam and actuator model}

Since the structure tested was thin with respect to its length, Euler beam theory was used to model the simply supported beam. This theory assumes that the rotary inertia of the beam and shear deformation is negligible. ${ }^{4}$ The equations for system response were modified to include structural damping by making the material stiffness complex. ${ }^{5}$ With these assumptions, Hamilton's extended principle can be used to develop the boundary-value problem. The resulting equation of motion of the structure is separable, and the eigenvalue problem can be readily solved.

The well-known solution for the simply supported beam is

$$
w(x, \tau)=\sum_{n=1}^{\infty} W_{n} \sin \left(\frac{n \pi x}{L_{x}}\right) e^{j \omega \tau} .
$$

Based on the expansion theorem, the system response to a harmonic input can be obtained in terms of the eigenvectors (modal response) of the system. Before developing the response of the beam, an approximate model of the input from the actuator must be developed.

\section{Analysis of actuator}

Piezoelectric elements are activated by applying a polarization voltage along their polarization direction. If an actuator is unconstrained, polarization in the $z$ direction will result in equally induced strains in the $x$ and $y$ directions of the actuator. The magnitude of this strain can be expressed as a function of the piezoelectric strain constant $d_{31}$, the applied voltage $V$, and the actuator thickness $t$ :

$$
\left(\epsilon_{x}\right)_{\mathrm{pe}}=\epsilon_{\mathrm{pe}}=\left(d_{31} / t\right) V,
$$

where

$$
d_{31}=180 E-12 \mathrm{~m} / \mathrm{V} \quad \text { (for G1195 material). }
$$

The subscripts pe and $b$ will be used when referencing properties of the piezoelectric material and beam, respectively. ${ }^{2}$

In developing the simplified theoretical model, the piezoelectric elements are assumed to be perfectly bonded to the structure with zero glue thickness. While a finite bonding layer will be encountered in practice, the negative effect of shear lag losses will be compensated to some extent by the increase in net moment loading as the shear stresses induced by the actuators are displaced from the neutral axis of the beam. In fact, previous work based on finite-element models of the glue layer, piezoelectric actuator, and structure indicate that a thin bonding layer results in a response identical to that of the case of zero glue thickness. In addition, results from finite-element analysis demonstrate that for thin bonding layers, material properties of the glue, such as the Young's modulus, have negligible effect on the structural response due to excitation by the piezoelectric actuator. ${ }^{6}$

Since the stiffness of the beam about the $x$ axis is 9.5 times greater than that about the $y$ axis and the length of the actuator in the $y$ direction is 2.3 times smaller than that in the $x$ direction, the bending of the beam about the $x$ axis will be negligible in comparison to that about the $y$ axis. As a result of this observation, the spatial response of the beam can be expressed simply as a function of the $x$ direction. The term actuator will apply to a patch pair, one bonded to the top of the structure surface and the other bonded to the bottom of the structure surface at the same coordinates. Since excitation of flexural waves is desired, when extension is induced in the piezoelectric element on the top surface, compression is induced in the corresponding element on the bottom surface, resulting in pure bending moments about the neutral axis of the beam. The following analysis follows directly from a previous analysis of a rectangular plate by Dimitriadis et al. ${ }^{2}$ as well as that of Crawley and de Luis. ${ }^{1}$

A schematic of the stress distribution is presented in Fig. $1 .{ }^{1,3}$ Since the actuators strain normal to the direction of polarization, a voltage applied in the $z$ direction will induce interface stresses and strains in the $x$ direction as well as in the $y$ direction. As a result, the schematic of Fig. 1 represents the assumed $x-z$ stress distribution at any point within the piezoelectric-beam structure. ${ }^{1}$ The equivalent bending moments can be obtained by integrating the actuator stresses.

The normal stress distribution can be reduced to couples per unit length as follows, where $y$ is into the paper (in Fig. 1):

$$
\int_{-h}^{+h} \sigma_{x} z d y d z=m_{x} d y .
$$

The strain distribution is linear and can be represented by

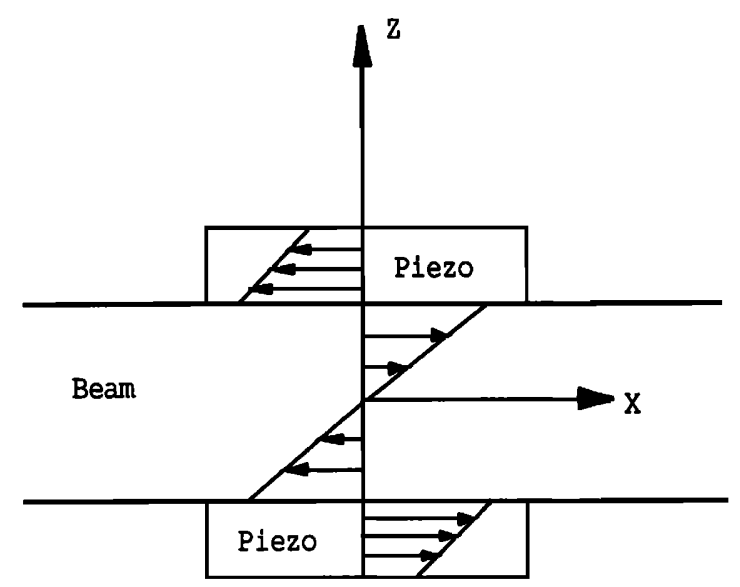

FIG. 1. Stress distribution. 


$$
\epsilon_{x}=z / r_{x},
$$

where $1 / r_{x}$ denotes the curvature of the neutral surface parallel to the $x-z$ plane at any point on the beam. By substituting Eq. (4) into Hooke's law and replacing the normal stress $\sigma_{x}$ with the couple $m_{x}$, the following expression can be obtained: ${ }^{1}$

$$
m_{x}=D\left(1 / r_{x}\right),
$$

where

$$
D=2 E_{b} h^{3} / 3 \text {. }
$$

Solving Eq. (6) for $1 / r_{x}$,

$$
1 / r_{x}=m_{x} / D \text {. }
$$

The actuator patch can be replaced by creating pure bending of the beam such that the bending surface of the beam is equal to the interface stress when the patch is activated.

To begin, the beam and piezoelectric patch interface stress-strain relations are derived directly from Hooke's law:

$$
\left(\sigma_{x_{i}}\right)_{b}=E_{b} \epsilon_{x_{i}} .
$$

By superimposing the external beam strains at the interface and unconstrained piezoelectric element strains, the actuator stresses can be derived:

$$
\left(\sigma_{x_{i}}\right)_{\mathrm{pe}}=E_{\mathrm{pe}}\left(\epsilon_{x_{i}}-\epsilon_{\mathrm{pe}}\right) .
$$

The bending stresses in the beam are linear in $z$ and can be written in terms of the interface values:

$$
\left(\sigma_{x}\right)_{b}=\left[\left(\sigma_{x_{1}}\right)_{b} / h\right] z .
$$

Similarly, the stresses in the piezoelectric element are

$$
\left(\sigma_{x}\right)_{\mathrm{pe}}=\left(\sigma_{x_{1}}\right)_{\mathrm{pe}}-\left(\sigma_{x_{1}}\right)_{b}(1-z / h) .
$$

After determining the interface stress of the beam, the uniformly distributed moments can be determined to produce the assumed linear stress distribution. However, the interface strains and beam-bending stresses must be derived in terms of the constituent material properties and unconstrained actuator strains since Eq. (8) is a function of the unconstrained piezoelectric strain and actuator interface strains.

Applying moment equilibrium about the neutral axis of the beam,

$$
\int_{0}^{h}\left(\sigma_{x}\right)_{b} z d z+\int_{h}^{h+t}\left(\sigma_{x}\right)_{\mathrm{pe}} z d z=0 .
$$

Substituting Eqs. (10) and (11) into Eq. (12) and evaluating the integral yields

$$
\left(\sigma_{x_{i}}\right)_{b}=\frac{3 t h(2 h+t)}{2\left(h^{3}+t^{3}\right)+3 h t^{2}}\left(\sigma_{x_{i}}\right)_{\mathrm{pe}} .
$$

For convenience, a nondimensional parameter $K$ is defined as follows:

$$
K=\frac{3 t h(2 h+t)}{2\left(h^{3}+t^{3}\right)+3 h t^{2}} .
$$

Equation (13) may now be substituted into Eqs. (8) and (9) to eliminate the interface beam strains from the expression:

$$
(1-P) \epsilon_{x_{1}}=-P \epsilon_{\mathrm{pe}},
$$

where

$$
P=-\left(E_{\mathrm{pe}} / E_{b}\right) K .
$$

Rearranging Eq. (14) yields the interface strain relation

$$
\epsilon_{x_{i}}=-[P /(1-P)] \epsilon_{\mathrm{pe}} .
$$

At this point, the interface strains have been reduced to a function of the unconstrained piezoelectric element strains and the constituent material properties. As a result, the uniformly distributed moments which produce the interface stress, $\left(\sigma_{x \neq A}\right)_{b}$, can be determined. Substituting the bendingcurvature-stress relationship into Eq. (5) yields

$$
m_{x}=\frac{2}{3} h^{2}\left(\sigma_{x_{1}}\right)_{b} .
$$

Substituting the interface-stress-piezoelectric-strain coupling relations Eqs. (8) and (17) into Eq. (18) yields the distributed surface moment:

$$
m_{x}=C_{0} \epsilon_{\mathrm{pe}},
$$

where

$$
C_{0}=-E_{b}[2 P / 3(1-P)] h^{2} .
$$

The previous formulation was based on the assumption of an infinite beam and piezoelectric element. Since in reality both are finite, a justification of the earlier assumption must be given. For a finite actuator patch pair, the normal stress distribution does not hold at the free edge where equilibrium conditions require the normal stress at the actuator boundary to be zero. However, Liang and Rogers ${ }^{7}$ showed that the actuator stress field for a distributed actuator is unaffected by the free edge up to approximately four actuator thicknesses from the boundary. As a result, if the actuator is large compared to its thickness, the assumed stress field depicted in Fig. 1 creates a case of pure bending in the beam, which is the fundamental premise of the formulation. Crawley and de Luis ${ }^{1}$ have also shown that when a finite piezoelectric strip is perfectly bonded to a beam, the induced moments effectively act at the element boundaries and result in pure bending of a one-dimensional structure. In the following derivation, the response of the simply supported beam due to excitation with a piezoelectric actuator will be derived.

\section{Excitation of simply supported beam}

After developing the piezoelectric-actuator-induced stress relations, the response of a finite beam due to excitation with a bonded rectangular actuator is derived. The beam depicted in Fig. 2 is simply supported at the boundaries as previously noted. To maintain consistency with the model, the actuator consists of two piezoelectric elements bonded symmetrically to the beam. Activation of the piezoelectric actuator will induce internal moments across the patch, and the strains are the same in both the $x$ and $y$ directions. Since the model for the beam is one dimensional, the strain induced in the $y$ direction will not be considered. This strain serves to increase the local stiffness of the beam about the $x$ axis due to the curvature induced. Since the stiffness about the $x$ axis is much greater than that about the $y$ axis, the curvature is small in comparison. To consider the effect of strain induced in the $y$ direction, a two-dimensional model of the structure is required. However, the purpose of this study is to evaluate the one-dimensional model by comparing modal amplitudes obtained from experiments and theory. 


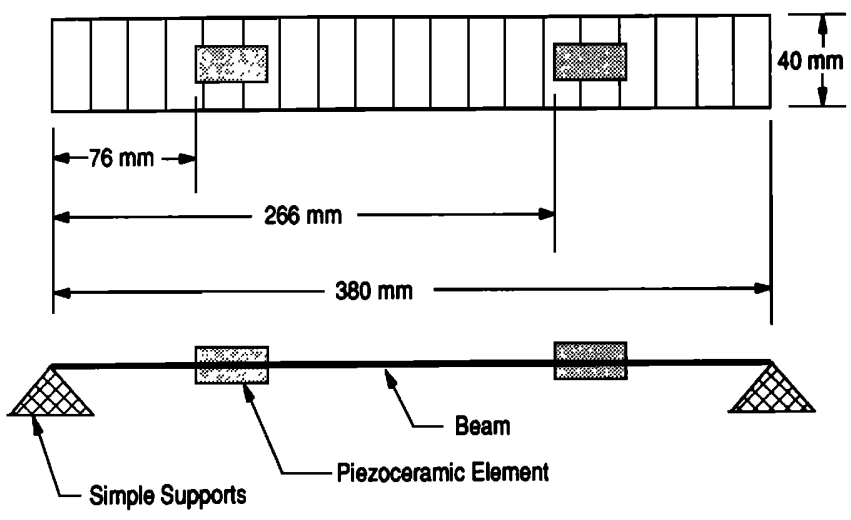

Beam thickness: $2 \mathrm{~mm} \quad$ Piezocerarnic dimensions: $16 \mathrm{~mm} \times 38.1 \mathrm{~mm} \times 0.2 \mathrm{~mm}$

FIG. 2. Simply supported beam.

The moment in the beam can be written in terms of the unit-step function where $x_{1}$ and $x_{2}$ indicate the spatial location of the actuator. In addition, since the actuator does not extend across the full width of the beam, the ratio of the width of the actuator to that of the beam will be used to scale the moment. By scaling the moment, the stress induced by the local strain of the actuator is distributed over the full width of the beam. This is analogous to distributing a concentrated load over the surface of application. The moment $m_{x}$ has the units of moment per unit length about the $y$ coordinate:

$m_{x}=C_{0} \epsilon_{\mathrm{pe}}\left[u\left(x-x_{1}\right)-u\left(x-x_{2}\right)\right]\left(w_{\mathrm{pe}} / w_{b}\right)$.

By oscillating the voltage across the actuator, the induced moment $m_{x}$ will oscillate at the same frequency. The equation of motion for the beam can be written due to excitation by the oscillating moment. The mass and stiffness loading on the beam due to the actuator are assumed negligible. The equation of motion is written in terms of the internal bending moment $M_{x}$ and the actuator-induced moment $m_{x}$ as follows:

$$
\frac{\partial^{2}\left(M_{x}-m_{x}\right)}{\partial x^{2}}+m^{\prime \prime} \ddot{w}=0,
$$

where $m^{\prime \prime}$ is the area mass density of the beam, and $w$ is the transverse displacement of the beam. ${ }^{5}$

The moment $m_{x}$ can be transferred to the right-hand side of Eq. (23), representing the external beam load. The internal moment $\boldsymbol{M}_{\boldsymbol{x}}$ can be expressed in terms of the displacement of the beam $w$, yielding the final form of the equation of motion:

$$
D \nabla^{4} w+m^{\prime \prime} \ddot{w}=\frac{\partial^{2} m_{x}}{\partial x^{2}},
$$

where $D$ is the flexural rigidity of the beam.

The external loads on the beam can be obtained by differentiating Eq. (21):

$\frac{\partial^{2} m_{x}}{\partial x^{2}}=C_{0} \epsilon_{\mathrm{pe}}\left[\delta^{\prime}\left(x-x_{1}\right)-\delta^{\prime}\left(x-x_{2}\right)\right]\left(\frac{w_{\mathrm{pe}}}{w_{b}}\right)$.

Substituting the above expression into the equation of motion yields

$$
\begin{aligned}
& D \nabla^{4} w+m^{\prime \prime} \ddot{w} \\
& \quad=C_{0} \epsilon_{\mathrm{pe}}\left[\delta^{\prime}\left(x-x_{1}\right)-\delta^{\prime}\left(x-x_{2}\right)\right]\left(w_{\mathrm{pe}} / w_{b}\right) .
\end{aligned}
$$

A moment $M$ acting upon a structure can be expressed as a dipole force with magnitude $M \delta^{\prime}\left(x-x_{0}\right)$, where $x_{0}$ is the location of the moment. Thus uniformly distributed reaction moments in the beam are the result of external line moments acting along the boundaries of the piezoelectric element.

The solution of Eq. (25) can be obtained by performing a modal expansion on the response previously outlined in Eq. (1). Substituting Eq. (1) into the equation of motion [Eq. (23)],

$$
\sum_{n=1}^{\infty}\left(\omega_{n}^{2}-\omega^{2}\right) W_{n} \sin \left(\frac{n \pi x}{L_{x}}\right)=\frac{1}{m^{\prime \prime}} \frac{\partial^{2} m_{x}}{\partial x^{2}},
$$

where

$$
\omega_{n}^{2}=D\left[(n \pi)^{4} / L_{x}^{4} m^{\prime \prime}\right] .
$$

As mentioned earlier, to include the effects of structural damping, the beam stiffness $E_{b}$ was defined to be complex. ${ }^{5}$ The resulting equation for modal amplitudes will yield finite results at a resonance which corresponds to results expected in real systems. Replacing the flexural rigidity in Eq. (27) by Eq. (6) and recalling that the structural stiffness is complex, $\omega_{n}^{2}$ can be written as

$$
\omega_{n}^{2}=\frac{2 E_{b}(1+i \eta) h^{3}(n \pi)^{4}}{3 L_{x}^{4} m^{\prime \prime}} .
$$

After applying the orthogonality condition, the modal amplitudes can be obtained:

$$
\begin{aligned}
W_{n}= & \frac{2 C_{0} \epsilon_{\mathrm{pe}} n \pi w_{\mathrm{pe}}}{\left(\omega_{n}^{2}-\omega^{2}\right) L_{x}^{2} m^{\prime \prime} w_{b}} \\
& \times\left[\cos \left(\frac{n \pi x_{2}}{L_{x}}\right)-\cos \left(\frac{n \pi x_{1}}{L_{x}}\right)\right] .
\end{aligned}
$$

The maximum modal amplitude will occur when the driving frequency is equivalent to the real part of Eq. (28). ${ }^{5}$ To obtain the modal amplitude resulting from excitation of multiple actuators, the superposition principle is invoked. Recalling Eq. (2),

$$
\left(\epsilon_{x}\right)_{\mathrm{pe}}=\epsilon_{\mathrm{pe}}=\left(d_{31} / t\right) V,
$$

we observe that the strain induced in the piezoelectric element is proportional to the voltage. To vary the modal response of the structure, the phase of the voltage between independent actuators can be varied, and the resulting modal amplitude can be computed in general for $m$ actuators:

$$
W_{n}^{m}=C V \sum_{k=1}^{m}\left[\cos \left(\phi_{k}\right)+j \sin \left(\phi_{k}\right)\right] f\left(x_{1_{k}}, x_{2_{k}}\right),
$$

where

$$
\begin{aligned}
& C=\frac{2 C_{0} d_{31} n \pi w_{\mathrm{pe}}}{\left(\omega_{n}^{2}-\omega^{2}\right) t L_{x}^{2} m^{\prime \prime} w_{b}} \\
& f\left(x_{1_{k}}, x_{2_{k}}\right)=\left[\cos \left(\frac{n \pi x_{2_{k}}}{L_{x}}\right)-\cos \left(\frac{n \pi x_{1_{k}}}{L_{x}}\right)\right] .
\end{aligned}
$$

After obtaining the final expression for the modal amplitudes, a summary of the previous assumptions is in order. The piezoelectric patch is assumed to be perfectly bonded to 
the beam, resulting in equivalent interface strains in the actuator and beam. As outlined by Crawley and de Luis, ${ }^{1}$ significant thickness of the bonding layer can render this assumption invalid. In addition, the piezoelectric element is assumed to have negligible effect on the inertial mass and stiffness of the beam. This assumption will certainly be dependent on relative size of the actuator compared to that of the structure; however, for commercially available piezoelectric elements with a thickness on the order of $0.2 \mathrm{~mm}$ and beams of the order of $2 \mathrm{~mm}$, the assumption is justified.

\section{EXPERIMENTAL DISCUSSION}

After developing the theoretical predictions for the modal response of the structure, a simply supported beam was designed and built. The theory to experimentally investigate the modal response of the structure as well as the range of linearity of response was developed.

\section{A. Experimental setup}

The experimental setup focused around the test and evaluation of the piezoelectric actuators bonded to a simply supported steel beam shown in Fig. 2. This beam was attached to light-gauge steel supports at the end points, and these supports were attached to a rigid steel structure. The ends of the beam were fixed spatially in the $z$ direction; however, the beam was relatively free to rotate about its ends, creating the desired simply supported boundary conditions. The beam itself was made of plain carbon steel. It is $380 \mathrm{~mm}$ in length, $40 \mathrm{~mm}$ wide, and $2 \mathrm{~mm}$ thick. Eighteen measurement locations are identified as shown, with the first location being the first interior line measured from the left side of the figure and the 18th location being the rightmost interior point. These measurement locations were referenced from the center of the beam and spaced at even $20-\mathrm{mm}$ intervals. The measurement locations were chosen such that the structure could be mapped for the modal analysis. In addition, discrete grid points provide a means of studying the effects of measurement location on the response of the structure.

The piezoelectric strips are referenced as G1195 material with dimensions of $38.1 \mathrm{~mm} \times 15.8 \mathrm{~mm} \times 0.2 \mathrm{~mm}$. Mbond 200 adhesive material was used to attach the actuators to the surface of the structure at spatial locations of 76 and $268 \mathrm{~mm}$ from the left end of the beam, as illustrated in Fig. 1. Before bonding the actuator to the structure, an insulating layer of glue is required to prevent shorting of the electrical contacts.

The piezoelectric actuators were driven with a signal generator configured with the B\&K 2032 signal analyzer. A power amplifier was used to boost the signal, and a transformer with a 17 to 1 ratio was used to increase the voltage necessary to drive the piezoelectric actuators. The input signal was measured prior to the transformer, and the output signal was obtained from a B\&K 4374 microaccelerometer configured with a B\&K type 2635 charge amplifier. Since the accelerometers weigh only $0.65 \mathrm{~g}$, mass coupling was assumed small. The signals were converted from analog to digital via the B\&K 2032 signal analyzer, and the data was transferred to a personal computer by an IEEE-488 instrumentation interface.
During all tests, the frequency response for the output signal was limited to $2000 \mathrm{~Hz}$ since higher frequencies do not provide significant additional information and limit the frequency resolution. In addition, the expected application for active vibration and noise control is most likely well below this frequency. A Hanning window was applied to the timedependent signal, and 64 averages were obtained for each complete data set with $50 \%$ overlap processing.

Several preliminary tests were conducted before proceeding with experiments devoted to studying distributed excitation of the structure. A modal analysis of the structure was performed to compare mode shapes and resonant frequencies from the designed structure and theoretical model. A discussion of the modal analysis is included in the Appendix. In addition, the range of linearity for the structural response must be identified before comparing results between experiments and theory. A description of this test and methods of analysis are also included in the Appendix.

\section{B. Distributed excitation and spatial decomposition}

The primary experimental test to be performed pertains to the topic of distributed excitation. Since the piezoelectric actuator is finite and must be bonded to the structure at some predetermined spatial coordinates, the possibility of driving the structure at any chosen mode was considered. To investigate this possibility, a spatial decomposition was performed. ${ }^{8}$ In other words, the piezoelectric device was driven at a constant amplitude and frequency, and the structural response was measured and decomposed into modal amplitudes. Two accelerometers were placed on the structure, with one of the accelerometers being the reference. The reference accelerometer was located at the first grid point such that all modes could be detected. The "floating" accelerometer was moved over eight grid points, allowing computation of eight modal amplitudes.

The frequency response function between the accelerometers was measured as well as the autospectrum of the reference accelerometer. The autospectrum provides a means of scaling the data in terms of engineering units. By computing the frequency response function, phase information is also obtained. If the beam is assumed to respond as predicted in the theoretical analysis from Euler beam theory, a matrix of spatial coefficients can be generated from the theoretical eigenvectors by substituting the spatial coordinates of the "floating" accelerometer into the equation. After repeating this for eight separate measurement locations, since eight distinct modal amplitudes can be computed, an $8 \times 8$ matrix of the eigenvectors will result. After measuring the acceleration at each of these coordinates, a system of linear algebraic equations results:

where

$$
[W]=[S][A]
$$

$$
\begin{gathered}
{[W]=\left[\begin{array}{c}
W_{1} \\
\vdots \\
W_{n}
\end{array}\right],} \\
{[A]=\left[\begin{array}{c}
A_{1} \\
\vdots \\
A_{n}
\end{array}\right],}
\end{gathered}
$$




$$
[S]=\left[\begin{array}{lllll}
S_{11} & S_{12} & S_{13} & \cdots & S_{1 j} \\
S_{21} & S_{22} & S_{23} & \cdots & S_{2 j} \\
S_{31} & S_{32} & S_{33} & \cdots & S_{3 j} \\
\cdots & \cdots & \cdots & \cdots & \cdots \\
S_{i 1} & S_{i 2} & S_{i 3} & \cdots & S_{i j}
\end{array}\right],
$$

where

$$
S_{i j}=\sin \left(j \pi x_{i} / L_{x}\right) .
$$

In the above equation, $j$ represents the mode number and $i$ represents the spatial position of the "floating" accelerometer for each data set. The modal accelerations were extracted by solving this system of linear equations. This method of obtaining the modal amplitudes should be contrasted to that of the typical modal approach. The standard approach in modal analysis is to obtain the experimental results from accelerometers placed at discrete spatial locations and compute frequency response functions (FRFs) between these measured outputs and the forcing inputs. The modal response is then obtained by curve fitting the experimentally obtained FRFs. The method utilized in this test can only be used when prior knowledge of the system response is known either analytically or experimentally; however, it enables the determination of modal amplitudes in off-resonance cases.

To determine if independent modes could be dominantly excited, the structure was driven at a variety of frequencies and phasing between the two actuators bonded to the beam. Some spillover is expected in other modes since the piezoelectric actuator is distributed over the surface of the structure. The goal is to determine the ability to excite independent modes of the structure. In Fig. 3 the orientation of the piezoelectric actuators can be seen with respect to the simply supported beam. The first six modes are depicted graphically to illustrate the relationship between the orientation of the actuator and nodal lines for each respective mode. For excitation of the second and third modes, the actuator lies in a region of significant curvature of the structure during vibra-

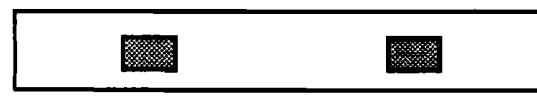

F1rst Mode

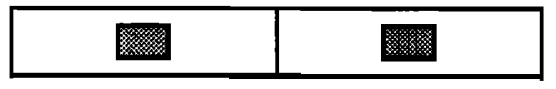

Second Mode

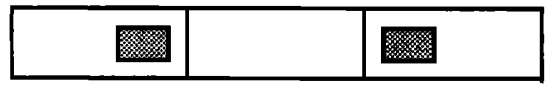

Third Mode

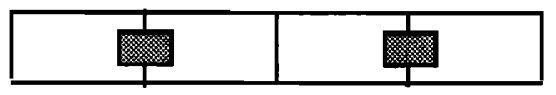

Fourth Mode

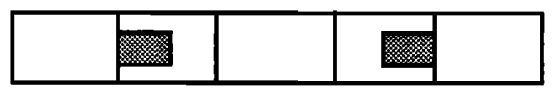

Fifth Mode

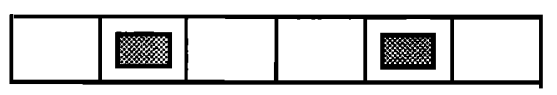

S1xth Mode tion. As a result, the actuator is expected to readily excite those modes of the structure. However, for the fourth mode of the simply supported beam, the center line of the actuator lies on the modal line. At this location, an inflection point would occur in the structure, resulting in a minimum concavity. Since the actuator essentially creates bending moments, this location conflicts with the coupling between the actuator and structure. Because of this conflict, spillover into adjacent modes is expected, with little or no excitation of the fourth mode.

\section{RESULTS}

A modal analysis of the beam was initially performed to determine if the assumption of Euler beam theory was justified in modeling the beam. Upon completing the modal analysis, the linearity test was studied to determine an appropriate working range of input voltages for the piezoelectric actuators. Finally, a test was performed such that experimental and theoretical results could be compared.

\section{A. Modal analysis and theoretical analysis}

The experimental modal analysis was performed to determine if the structural response exemplified a simply supported beam. For comparison, the theoretical eigenvalues and eigenfunctions for the experimental beam were computed. Common material properties for steel were chosen with a Young's modulus of $29 \mathrm{Mpsi}(200 \mathrm{GPa}$ ) and a density of 487 $\mathrm{lb} / \mathrm{ft}^{3}\left(7800 \mathrm{~kg} / \mathrm{m}^{3}\right)$. The modal analysis of the structure yielded 13 eigenvalues, 6 of which are presented in Table $I$.

Tabulated with the experimental results are the theoretical predictions for the first six resonant frequencies of the structure. It should be noted that the theoretical model includes a term for structural damping; however, this term simply serves to create a finite response at resonance. For the first four modes of the structure, the predicted values of the resonant frequencies are less than the experimentally measured values; however, for modes 5 and 6 , the predicted values exceed the measured values. In both cases, the percent difference in predicted and measured values for resonant frequencies is on the order of $3 \%$.

The first four resonant frequencies obtained experimentally are observed to be higher estimates of the theoretical resonant frequencies. This can be explained by examining the spectral resolution. Since the base bandwidth was 2000 $\mathrm{Hz}$ and 400 spectral lines were displayed, the spectral resolution was $5 \mathrm{~Hz}$. For the first mode, the resonant frequency was estimated between the discrete spectral lines of 30 and

TABLE I. Theoretical versus experimental resonant frequencies.

\begin{tabular}{crc}
\hline \hline Mode & Theoretical $f_{n}(\mathrm{~Hz})$ & Experimental $f_{n}(\mathbf{H z})$ \\
\hline 1 & 31.1 & 32.9 \\
2 & 124.4 & 126.9 \\
3 & 280.0 & 282.3 \\
4 & 497.7 & 498.6 \\
5 & 777.7 & 768.7 \\
6 & 1119.9 & 1077.0 \\
\hline \hline
\end{tabular}

FIG. 3. Modes of beam with respect to actuators. 


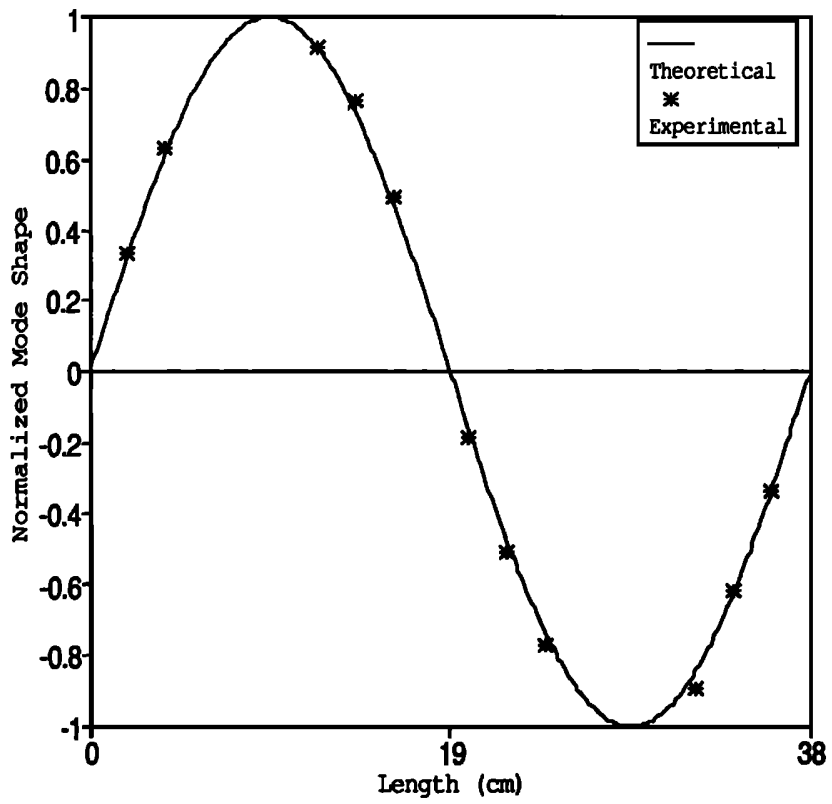

FIG. 4. Theoretical mode shape versus experimental data.

$35 \mathrm{~Hz}$, resulting in poor resolution. To more accurately analyze the lower modes, a smaller base bandwidth could be chosen or zoom processing could be imposed. However, since the goal of this study was to compare results primarily for operating conditions other than resonance, results obtained from the modal analysis were sufficient to proceed.

The curve-fitting routine also yielded estimates of the eigenvectors for each respective mode. The theoretical and experimental eigenvectors were normalized, and the mode shapes for the first six modes were plotted from the theoretical and experimental analysis and superimposed on each other. An example is presented in Fig. 4. The experimental and theoretical modes compare well for the first five modes; however, some deviation was noted at the sixth mode. This deviation results from the fact that contributions of higher modes, above the $2000-\mathrm{Hz}$ frequency range, are not available for curve fitting.

For the goals outlined in this study, the structural response can be considered to follow that of a simply supported beam as desired. While this lends confidence to analysis of the remaining results, it was essential for performing the spatial decomposition based on the theoretical eigenfunctions.

\section{B. Linearity}

The results of the linearity tests demonstrated that the piezoelectric material excited the beam in a linear manner for input voltage levels ranging from 5 to $75 \mathrm{~V} \mathrm{rms}$ over the $2000-\mathrm{Hz}$ range. The results have been quantified with a frequency response plot for the acceleration measured at the first grid point as described earlier. For $95 \%$ confidence intervals on the magnitude of the FRF, the separation between the upper and lower confidence bounds was on the order of $0.4 \mathrm{~dB}$. To clarify the plot, only the average of the frequency response functions was plotted, realizing that all of the FRFs were within the computed confidence interval. Refer to Fig.

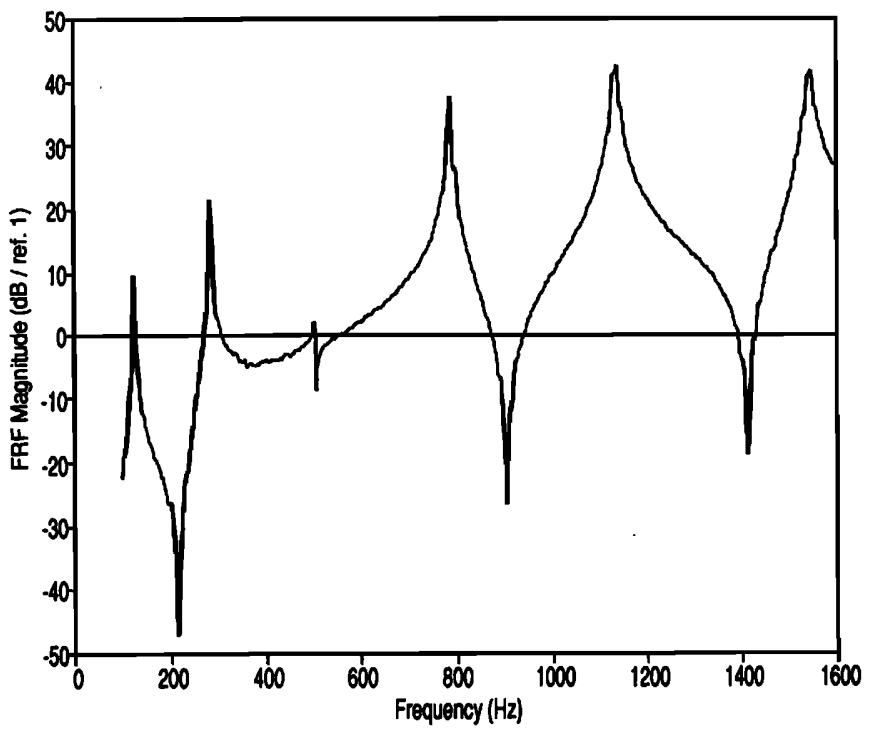

FIG. 5. Average frequency response function of simply supported beam.

5. Large deviations in the response were noted at frequencies below $100 \mathrm{~Hz}$; however, coherence was poor in this region because of the inability of the piezoelectric actuator to respond efficiently in this operating range. To clarify the results, plotting of the data in this regime was suppressed.

Results from a statistical analysis of the phase were eliminated since they essentially replicated that of the magnitude in linearity. As previously mentioned, a $95 \%$ confidence interval was chosen as the criterion for determining the range of linearity. This confidence interval is based upon the normalized error of the FRF, which, in turn, is dependent upon the coherence. Before continuing, a few notes about the coherence are worthy of mentioning. The coherence of the output to the input was on the order of 0.95 , except at resonance where the coherence dropped significantly. This can be expected since the force decreases on resonance, reducing the signal-to-noise ratio. This problem could be eliminated by implementing burst random excitation. The separation of the confidence intervals were on the order of $1.2 \mathrm{~dB}$ at both resonance and antiresonance. The large separation at antiresonance is due to low output from the structure. Based on this knowledge, the response of the structure in the frequency range tested can be considered linear for the input levels previously documented.

After completing the test with a bandlimited input voltage, a harmonic input voltage was used to drive the structure. The frequency response function was measured as previously described; however, the only frequency of interest was the driving frequency. Harmonic inputs at frequencies of $128,200,278,400$, and $500 \mathrm{~Hz}$ were utilized to drive the structure, and the voltage was varied from approximately 15 to $150 \mathrm{~V} \mathrm{rms}$ in $15-\mathrm{V}$.increments. The response of the structure was linear in voltages ranging from 30 to $90 \mathrm{~V}$ rms applied across the actuator. A 5\% rise in the frequency response function was noted from the 15-30-V rms range, while a 5\% decrease in the FRF was noted in the 90-150-V rms range.

Results from the linearity tests indicate that the struc- 
tural response is linear under typical operating voltages and deviations from linear response are on the order of $5 \%$ for higher voltage levels. Since only discrete frequencies were tested with harmonic inputs, the results from the bandlimited input can be used to infer that the linearity measured at these frequencies is typical for inputs in the $100-2000-\mathrm{Hz}$ frequency range for voltage levels ranging from 30 to $90 \mathrm{~V}$ rms.

\section{Distributed excitation and spatial decomposition}

To study the effects of distributed excitation, the piezoelectric actuator was driven at various harmonic frequencies on and off resonance. The goal was to determine the ability of the actuator to excite various modes of the structure and examine the effects of spillover. For the purpose of this study, spillover will be used to describe the response of undesired modes due to the chosen spatial location of the actuators on the structure, and the undesired modes will be termed residual modes. In other words, relative phasing of voltage between actuators can be chosen to elicit the response of a desired mode; however, because of the location of the actuators on the structure, other residual modes will contribute to the overall response. A variety of tests were conducted to compare the predicted and measured response of the desired modes and residual modes of the structure due to harmonic excitation of piezoelectric actuators.

Since the theoretical and experimental eigenfunctions compared well, the spatial matrix of eigenvectors utilized in forming the system of linear algebraic equations was used to determine the modal amplitudes at a given frequency from the displacement data. The actuators were driven both independently and coupled. During the first series of tests, the leftmost actuator was driven at frequencies of 100,200 , and $350 \mathrm{~Hz}$. Following this test, the actuators were driven simultaneously both in phase and out of phase at frequencies of $100,150,200$, and $350 \mathrm{~Hz}$. Since the goal of the study was to compare the theoretical response of the structure to the experimental response, a computer program was written to predict the modal amplitudes for the above chosen tests from Eq. (38).

Results from excitation of the leftmost actuator at frequencies of $200 \mathrm{~Hz}$ are presented in Fig. 6. Since this frequency of excitation lies between the second and third mode of the structure, both theoretical and experimental results indicate structural response at the corresponding modes. Results are plotted in units of modal amplitude, which can readily be obtained from the modal acceleration for harmonic excitation by multiplying the modal acceleration by the square of the circular frequency. Results from excitation of a single actuator at 100 and $350 \mathrm{~Hz}$ were not as interesting since they simply illustrated dominant response of the structure at the second and third modes, respectively. As illustrated in Fig. 6, the theoretical response of the structure at the fourth mode was negligible. This result is due to the location of the piezoelectric actuator on the structure. When an actuator is excited at a given frequency, it essentially responds by bending at that frequency. This motion is opposed to the motion of the structure in the region of a nodal line since an inflection of the structure is observed at this location. Spill-

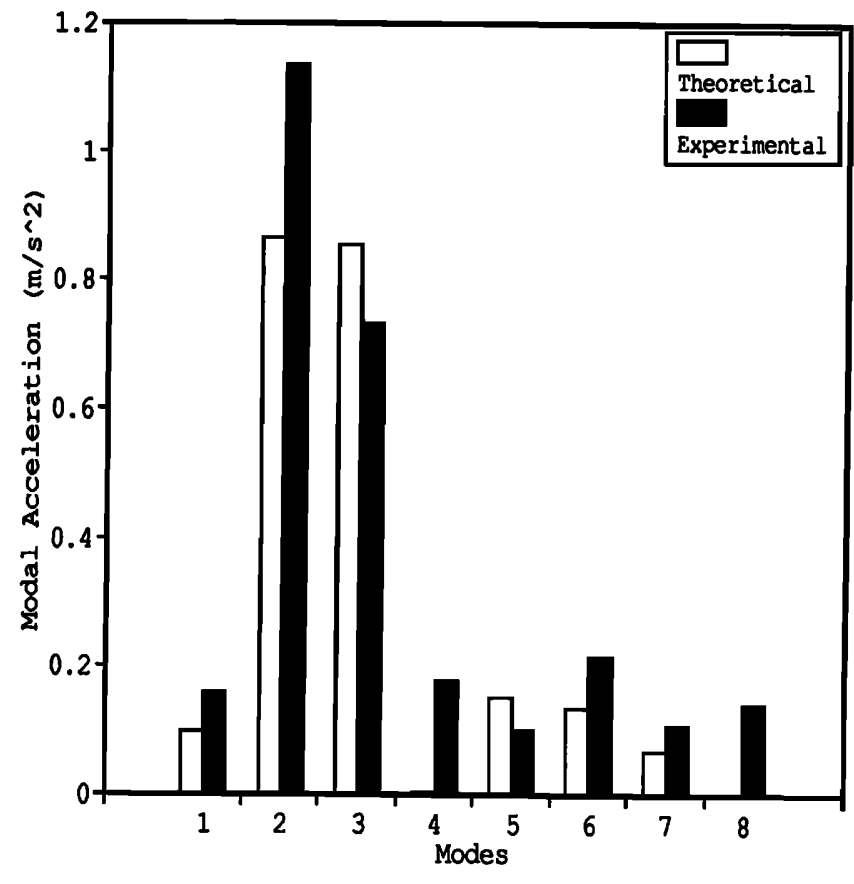

FIG. 6. Modal response versus mode number (patch pair 1, $200 \mathrm{~Hz}$ ).

over into adjacent modes occurs where the response of the structure is better aligned with the motion of the actuator.

In contrast to the theoretical results presented in Fig. 6, experimental results indicate some response at the fourth mode; however, these results are subject to errors due to the truncation of higher modes in solving for the modal amplitudes (i.e., spatial aliasing). The error due to truncation of higher modes is assumed small since the structure was driven by a single harmonic voltage. However, in view of this problem, the computed response of modes on the order of 20 dB lower than the response of the dominant modes is suspect.

For the case of distributed excitation, results from tests conducted at $100 \mathrm{~Hz}$ with actuators out of phase and $150 \mathrm{~Hz}$ with actuators in phase are presented in Figs. 7 and 8, respectively. When exciting the two actuators out of phase at 100 $\mathrm{Hz}$, the second mode of the structure is dominant in the response. By driving the actuators $180^{\circ}$ out of phase, the motion of the actuators correspond to that of the second mode of the structure, which can be seen by referring to Fig. 3. The same actuators were then driven in phase at $150 \mathrm{~Hz}$. This excitation frequency is nearest the second mode; however, the dominant response of the structure was at the third mode. This result was confirmed both experimentally and theoretically, illustrating that selection of location and phasing of the actuators is as important as the choice of the excitation frequency in driving the structure at a desired mode. Another observation worthy of mention is the response of the structure at the first mode for each of the previous test cases. When the actuators were driven in phase, the first mode contributed significantly to the structural response. However, when driven out of phase, the contribution of the first mode was suppressed, thus demonstrating control over residual modes of the structure. 


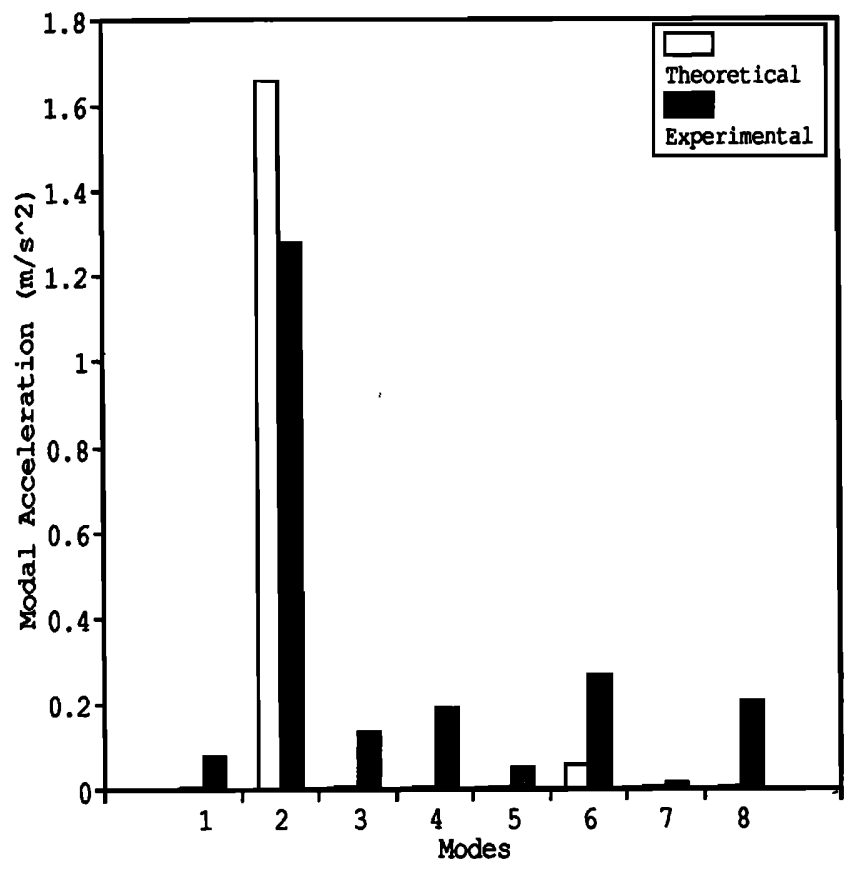

FIG. 7. Modal response versus mode number (patch pairs 1 and 2, out of phase at $100 \mathrm{~Hz}$ ).

Further indication of the importance of phasing was observed when driving the actuators at $200 \mathrm{~Hz}$. When driven in phase, the structural response was greatest at the third mode as seen in Fig. 9. In Fig. 10 results for excitation of the actuators out of phase are presented at the same frequency. The second mode of the structure was dominant in response for this configuration, and the remaining modes were sup-

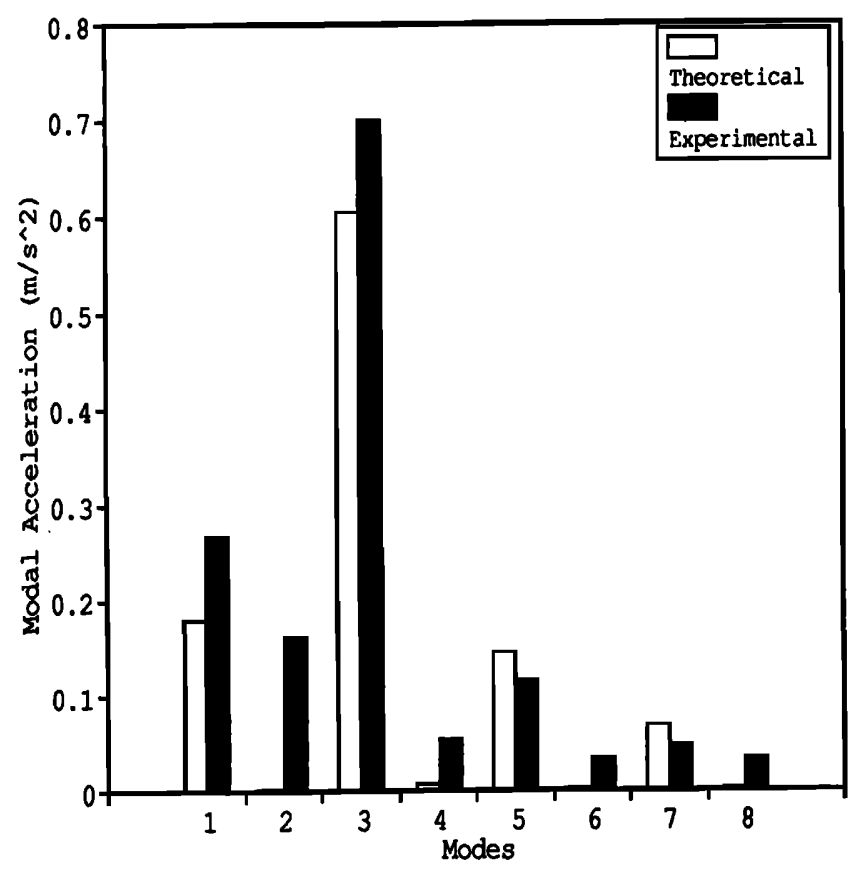

FIG. 8. Modal response versus mode number (patch pairs 1 and 2, in phase at $150 \mathrm{~Hz}$ ).

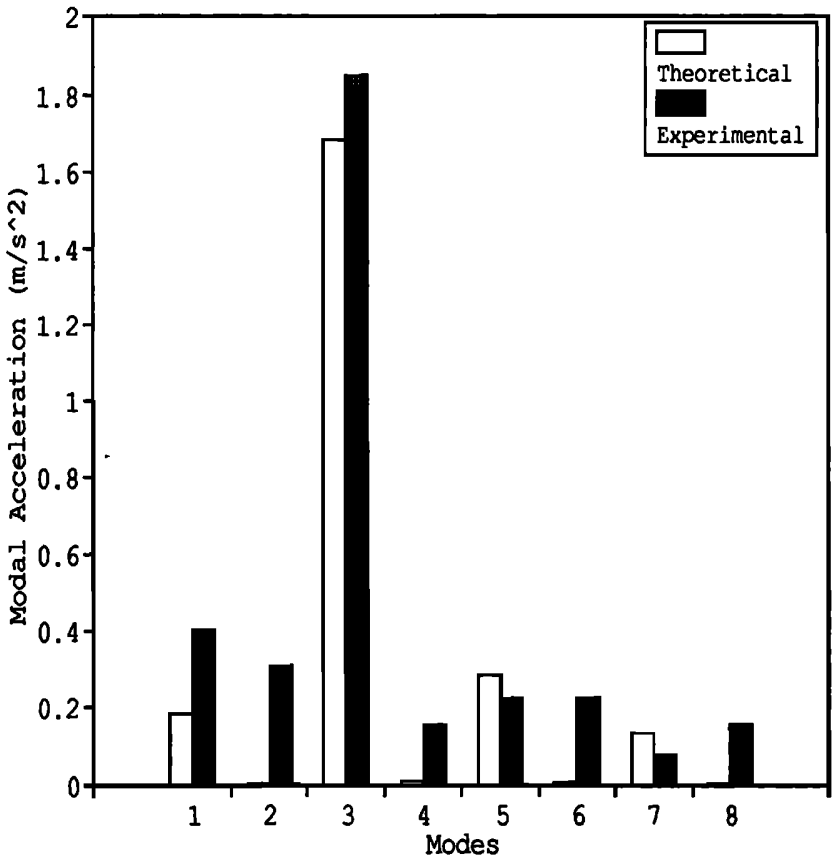

FIG. 9. Modal response versus mode number (patch pairs 1 and 2, in phase at $200 \mathrm{~Hz}$ ).

pressed. Again, these results are clarified upon referring to Fig. 3, which illustrates the position of the actuator in reference to the modes of the structures. Comparing the results of Figs. 9 and 10 to those of Fig. 6 again demonstrates that not only can the dominant mode be affected with distributed excitation, but also the residual modes. In Fig. 6 it is apparent that both the second and third modes respond strongly.

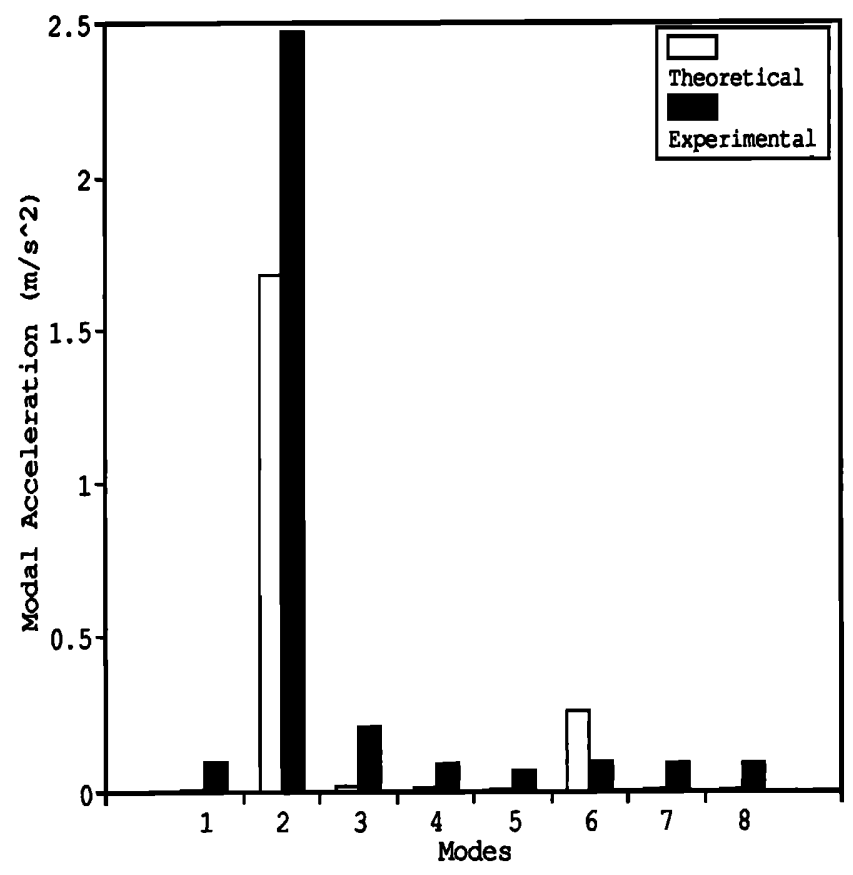

FIG. 10. Modal response versus mode number (patch pairs 1 and 2, out of phase at $200 \mathrm{~Hz}$ ).

Clark et al:: Multiple piezoelectric actuators 
When two in phase actuators are used, the response switches to the third mode while the second mode is suppressed. When two out of phase actuators are used, the second mode is dominant while the residual (third) mode is suppressed.

From the results presented, it is apparent that implementation of a greater number of actuators will lead to increased excitation of selected modes with reduced spillover. In fact, the ideal actuator for steady-state excitation would be, as shown by Meirovitch and Norris, ${ }^{9}$ an infinite number of infinitely small independently controlled elements completely covering the beam. In this way, a generalized control force can be created which is orthogonal to all modes except that which is required to be excited. In summary, phasing of the actuators in achieving excitation of chosen structural modes was shown to be as significant as choice of excitation frequency. In addition, the theoretical and experimental results were, on the average, within $25 \%$ of each other for the dominant modes of the structure. Differences in experimental and theoretical results were thought to be due to a number of factors, of which the most important were likely to be finite bonding layer, variation in the $d_{31}$ constant, asymmetry of actuator bonding and position, and errors inherent in the modal decomposition procedure.

\section{CONCLUSIONS}

A theoretical and experimental study of the nature of multiple piezoelectric patches as a distributed actuator has been made. A number of important conclusions have been obtained.

(1) The analytical model for the response of the simply supported beam to a single piezoelectric excitation source gave predictions which were within $25 \%$ of experimental results for a wide range of frequencies. The distribution of residual modes was also well predicted. Thus the analytical model has been largely confirmed and is useful in determining optimal actuator configurations for one-dimensional systems.

(2) Likewise, the response of the beam to multiple independently driven patches was also well predicted. In particular, the use of multiple independent actuators in selectively exciting required modes as well as suppressing unwanted residual modes was demonstrated. The model is thus useful in designing arrays of multiple independently driven piezoelectric actuators in order to selectively excite modes with control over the residual response.

(3) The response of the simply supported beam to excitation by piezoelectric actuators employed in this experimental test was demonstrated to be linear over a wide range of input voltages from 30 to $90 \mathrm{~V}$ rms with only a $5 \%$ deviation in the 90-150-V rms range. Excitation of the structure with piezoelectric elements below $50 \mathrm{~Hz}$ was found to be difficult.

Discrepancies between the modal amplitudes predicted from theory and those resolved from structural measurements are likely the result of several assumptions made in the analysis. Since a one-dimensional equation was used to predict the response of the simply supported beam, the effective increase in stiffness of the beam due to strain in the $y$ direction was not included in the model. In addition, since the piezoelectric actuators did not span the full width of the beam, the induced moment was scaled by the ratio of the width of the actuator to that of the beam. This is analogous to distributing a concentrated load over the surface of application. Finally, in the experimental analysis, a finite number of structural measurements were taken, limiting the number of modes which can be resolved computationally. As a result, spatial aliasing of the unresolved higher modes possibly contributed to the noted discrepancies between theoretical and experimental results for the residual modes.

If the goal is to predict a more accurate response of the beam, a finite-element analysis is in order, including the structural response in the $x$ and $y$ directions. However, for the purpose of choosing optimal actuator locations and relative structural response for a given control application, the one-dimensional model is more than sufficient. Even if the goal is to more accurately predict the response of the beam, a model such as that presented is best suited for initial studies to determine optimal actuator locations for eliciting the desired beam response since the computational time required is minimal compared to that required by finite-element analysis.

\section{ACKNOWLEDGMENTS}

This work was supported by ONR/DARPA under Grant No. ONR-N00014-88-K-0721.

\section{APPENDIX}

\section{Modal analysis}

Before attempting to characterize the relationship between the piezoelectric actuator and the structure, a modal analysis of the simply supported beam was performed. A grid was sketched on the beam as depicted in Fig. 2. An accelerometer was located at the first grid point since this is close to the support in a region where all of the modes within the desired frequency range could be measured. The accelerometer was used to measure acceleration, and an impact hammer was used to supply the forcing input. The structure was tapped at all of the grid points except at locations where piezoelectric actuators were bonded to the structure. The measured frequency range was from 0 to $2000 \mathrm{~Hz}$. Frequency response functions between the reference point (location of the accelerometer) and the impact locations were obtained, totaling 12 independent data sets. A series of data sets are required, including the point mobility of the excitation position to obtain an estimate of the eigenvalues and eigenfunctions of the structure.

Each data set was saved and a computer program implementing a Forsythe orthogonal polynomial to curve fit each FRF was used to perform the modal decomposition. ${ }^{10}$ From this program, the modal parameters, resonant frequencies, and corresponding damping coefficients were obtained. The program ultimately improves the estimate of the modal parameters by reducing the impact of the fit of the data in regions of poor coherence. ${ }^{10}$ The curve fit results in a numerator polynomial and a denominator polynomial for each FRF. The denominator of each curve fit should be consistent since this is essentially the characteristic equation of the 
structure, containing the natural frequencies and damping coefficients. The numerator contains information necessary to extract the mode shapes. After curve fitting all of the frequency response functions, the results can be combined and the corresponding mode shapes are computed.

\section{Linearity test}

The linearity of the strain applied by the piezoelectric material may be characterized by examining the response of a driven structure for a variety of voltage levels applied to the piezoelectric material. If the response of the structure differs at different voltages levels by a constant of proportionality, the system may be considered linear. The response of the structure can be characterized by the FRF. The FRF represents the ratio of the output response to input response for a system over some range of frequencies. Therefore, a linear system should have the same FRF for all input voltage levels. If an increase of the input amplitude above a certain level causes the FRF to deviate too far (this must be specified statistically) from the mean path, the nonlinear threshold can be identified.

The first step in determining the FRF of a system is recording the input and output levels of the system. An easy way to make sense of the system input and output records is to convert the time histories to the frequency domain using the Fourier transform. Generally, a model for the FRF is created based upon the assumption that there is an uncorrelated noise content disturbing the measurement of either the input or output. Because this noise is not correlated with the true output or input, the expected value of the product of the FFT for the output or input with this noise over a large number of averages is zero. The $H_{1}$ model was used to estimate the FRF, assuming noise on the output only, which will suffice for this experiment:

$$
H_{1}(j \omega)=G_{x y}(j \omega) / G_{x x}(j \omega) .
$$

By using $H_{1}(j \omega)$ and ignoring the possibility of noise on the measurement of the input signal, the results could be biased. However, the amount that the FRF is biased should not change with the variance of the input signal because it is assumed that the noise is uncorrelated to the output (or input). Thus, when increasing the variance of the input signal produces a value of $H_{1}(j \omega)$ that deviates significantly from values previously calculated for smaller input variances at a given frequency, the system response will be assumed nonlinear. ${ }^{11}$

While the $H_{1}$ model achieves adequate noise reduction on the output, it is necessary to determine what content of the measured signal is noise in order to obtain a good statistical estimate of $H_{1}(j \omega)$. With the assumption of uncorrelated noise,

$$
\begin{aligned}
& G_{x x}=G_{u u}+G_{n n}, \\
& G_{y y}=G_{v v}+G_{m m}, \\
& G_{x y}=G_{u v} .
\end{aligned}
$$

The measure of noise content is the coherence; its value approaches zero for large noise content and one for zero noise content:

$$
\gamma_{x y}^{2}(j \omega)=\frac{G_{x y}(j \omega) G_{y x}(j \omega)}{G_{x x}(j \omega) G_{y y}(j \omega)},
$$

where

$$
0 \leqslant \gamma_{x y}^{2}(j \omega) \leqslant 1 .
$$

A confidence interval for the FRF can be calculated based on the normalized rms error and Student's $t$ test for large data sets. The normalized error for the frequency response function is ${ }^{11}$

$$
\epsilon\left[H_{1}(j \omega)\right]=\frac{\left[\gamma_{x y}^{2}(j \omega)\right]^{2}}{\gamma_{x y}(j \omega) \sqrt{2 n_{d}}}
$$

A 95\% confidence interval was used to determine bounds for significant variation of the calculated values:

$$
\begin{aligned}
\hat{H}_{1}(j \omega)(1-1.96 \epsilon) & \leqslant H_{1}(j \omega) \\
& \leqslant \hat{H}_{1}(j \omega)(1+1.96 \epsilon) .
\end{aligned}
$$

With a means of evaluating linearity, the test can be performed. For this study, the input is created with both a random noise generator and a harmonic oscillator. The input voltage to the piezoelectric actuator was measured since it is assumed proportional to the forcing function. The output is obtained by placing an accelerometer on the beam at the first nodal point. All signals were filtered with an antialiasing filter, and the time histories were windowed with a Hanning window to reduce the uncorrelated content. By utilizing random noise as an input, a broadband of frequencies can be characterized with each test; however, the input must be characterized by a variance on the bandlimited noise applied. To gain a better understanding of the range of linearity, a harmonic oscillator is also used to excite the piezoelectric actuator at various frequencies in the range of the random noise generator. By using a harmonic input, a threshold of input voltage for linearity can be identified as opposed to a variance of input voltage.

\footnotetext{
' E. F. Crawley and J. de Luis, "Use of piezoelectric actuators as elements of intelligent structures," AIAA J. 25, 1373-1385 (1987).

${ }^{2}$ E. K. Dimitriadis, C. R. Fuller, and C. A. Rogers, "Piezoelectric actuators for distributed noise and vibration excitation of thin plates," in Proceedings of ASME 8th Biennial Conference on Failure Prevention and Reliability, Montreal, Canada (ASME, New York, 1989), pp. 223-233.

${ }^{3}$ J. L. Fanson and J. C. Chen, "Structural control by the use of piezoelectric active members," in Proceedings of NASA / DOD Control-Structures Interaction Conference, Rep. No. NASA CP-2447, 1986 (unpublished), Pt. II.

${ }^{4}$ L. Meirovitch, Elements of Vibration Analysis (McGraw-Hill, New York, 1987).

${ }^{5}$ L. Cremer, M. Heckl, and C. A. Ungar, Structure-Borne Sound (Springer, Berlin, 1973).

${ }^{6} \mathrm{M}$. R. Flemming, "An experimental investigation of the harmonic excitation of simply supported plates with surface-bonded piezoceramic actuators," VPI\&SU Master of Science thesis, Department of Mechanical Engineering, March 1991.

${ }^{7}$ C. Liang and C. A. Rogers, "Behavior of shape memory alloy actuators embedded in composites," in Proceedings of the 1989 Composites Conference, Beijing, China, August 1989 (unpublished).

${ }^{8}$ C. H. Hansen, S. D. Snyder, and C. R. Fuller, "Noise reduction of a vi-
} 
brating square panel by use of active sources and active vibration sources: A comparison," in Proceedings of Noise and Vibration 89, Singapore, Rep. No. E50-E57, 1989 (unpublished).

${ }^{9}$ L. Meirovitch and M. A. Norris, "Vibration control," in Proceedings of Inter-Noise 84, 1984 (unpublished), pp. 477-482.
${ }^{10}$ M.-C. Han, "On the application of Forsythe orthogonal polynomials for global modal parameter estimation," in Proceedings of the Seventh International Modal Analysis Conference, 1989 (unpublished), Vol. 1, pp. 625-630.

${ }^{11}$ J. S. Bendat and A. G. Piersol, Random Data (Wiley, New York, 1986). 